\title{
Comparative Evaluation of Neuromorphological Effects Under Single and Fractionated Irradiation in Small Doses
}

\author{
Ushakov IB*, Fedorov VP, Komarevtsev VN and Dyachkov AA \\ Federal Medical Biophysical Centre Moscow, Russia
}

Received: 眥 January 14, 2019; Published: 眥 January 18, 2019

*Corresponding author: Ushakov IB, Federal Medical Biophysical Centre Moscow, Russia

\section{Opinion}

During the experiment, 180 mongrel male rats at the age of 4 months (corresponding to approximately 27-28 human years) were irradiated with $\gamma$-quanta 60Co with an energy of $1.2 \mathrm{MeV}$ single and fractionated (in uniform portions for 5 days) in total doses of $50 \mathrm{cGy}$ at a dose rate of $50 \mathrm{cGy} / \mathrm{h}$. Neuromorphological changes of the sensorimotor cortex of the cerebral hemispheres in the III and $\mathrm{V}$ layers (respectively small and large pyramidal neurons) were studied. The material was taken in the first hours and days $(1,3$, $7,14)$ and also in $1,3,6,12,18$ and 24 months after the affected period. Each group had adequate age control. During the analysis of the study results, the main attention was paid to such radiation targets as protein and nucleic acids. The structural and functional rearrangement of neurons- as well as the state of enzymatic systems, neuroglia and microcirculatory channel - was assessed by tinctorial and morphometric parameters. Dispersion analysis was used to compare neuromorphological parameters under single and fractionated irradiation in the early (1 day) and late (18 months) periods of the affected period.

A day later, the number of neurons with functional changes (hypo- and hyperchromic) among small pyramidal neurons depended on all modes of irradiation, but the most influence on the dynamics of the indicator had a fractionated effect. The irradiation effect on the change in the functional activity of neurons is quite high: the significance level of the linear regression model is less than 0.0001 with the coefficient of determination R2=0.96 and strong correlation of arguments ( $r=0.98)$. Among the large pyramidal neurons, the number of neurons with functional changes depended only on single irradiation. The effect of irradiation on changes in the functional activity of neurons is high: the significance level of the model is less than 0.05 with the coefficient of determination R2=0.76 and strong correlation of arguments $(r=0.87)$. The number of destructive neurons depended on all modes of irradiation (significance level less than 0.05), but their dynamics was more strongly influenced by fractionated effects. The effect of fractionated irradiation on the dynamics of destructive neurons is high: the significance level of the model is less than 0.05 with the determination coefficient R2=0.70 and strong correlation of the arguments $r=0.84$.

The protein content in neurons depended on single irradiation, which is two times more affected the dynamics of the index compared to fractionated. The significance level of the model is more than 0.05 with a weak coefficient of determination $(\mathrm{R} 2=0.32$ ) and moderate correlation of arguments $(r=0.57)$. The content of RNA in the cytoplasm of neurons depended on all modes of irradiation, but the most influence on its dynamics had fractionated effect: the level of significance of the model is less than 0.001 with a coefficient of determination R2 $=0.69$ and a strong correlation of arguments ( $\mathrm{r}=0.83)$. The size of the neurons' nuclei depended on all of the exposure modes, but the greatest impact on their dynamics had single exposure. The significance level of the model is less than 0.0001 with the coefficient of determination R2=0.75 and strong correlation of arguments ( $r=0.87)$. The content of nuclear DNA depended on all irradiation modes, but the most influence on the dynamics of the indicator had fractionated effect: the level of significance of the model is less than 0.0001 with high diagnostic significance of the model $(\mathrm{R} 2=0.91)$ and a strong correlation of arguments ( $\mathrm{r}=0.96)$.

The content of RNA in nucleoli depended only on fractionated irradiation: the level of model significance is less than 0.0001 with the coefficient of diagnostic significance R2=0.88 and strong correlation of arguments ( $\mathrm{r}=0.94)$. The dynamics of the area of the cytoplasm of neurons was authentically dependent on single irradiation and only at a significance level of less than 0.3 . Thus - in the early periods of the affected period - in the III layer of 
the sensory-motor cortex of the brain fractionated irradiation had a more significant effect on the dynamics of the functional activity of neurons. Parameters of normochromic and destructive neurons did not depend on the irradiation regime. In the V layer, single irradiation had a greater impact on the dynamics of neurons with functional changes, reducing their number. Comparison of indicators of "normochromic and destructive neurons" at single and fractionated exposure did not reveal significant differences between them. Fractionated irradiation had a greater impact on the dynamics of RNA content in the cytoplasm and DNA in the nuclei, and single irradiation more influenced the size of the nuclei of neurons. After 18 months of the affected period among small pyramidal neurons the number of neurons with functional changes depended only on fractionated irradiation.

The significance level of the model is less than 0.05 with the coefficient of determination $\mathrm{R} 2=0.63$ and strong correlation of arguments $(\mathrm{r}=0.79)$. The number of destructive neurons depended on single irradiation, which is 4.4 times more strongly influenced the dynamics of this type of cells compared to fractionated. The effect of a single irradiation on the dynamics of the indicator is high: the level of significance of the model is less than 0.05 with a coefficient of determination $\mathrm{R} 2=0.73$ and the strong correlation case $(r=0.85)$. Among the large pyramidal neurons the number of neurons with functional changes (hypo- and hyperchromic) depended on single irradiation, which was 2.4 times stronger than the fractionated irradiation. The effect of single irradiation on the change in the functional activity of neurons is average: the significance level of the model is less than 0.05 with the coefficient of determination R2 $=0.37$ and moderate correlation of arguments $(\mathrm{r}=0.61)$. The number of destructive neurons depended on all modes of irradiation, but the fractionation had a stronger impact on the dynamics of the index compared to single irradiation. The significance level of the model is less than 0.05 with the coefficient of determination R2 $=0.58$ and strong correlation of arguments $(\mathrm{r}=0.76)$.

The size of the neurons' cytoplasm depended on a single irradiation, when the effect is twice stronger than the effect of fractionated irradiation. The effect of single irradiation on the dynamics of the sizes of the cytoplasm is not explicit: the level of significance of the model is less than 0.05 with a coefficient of determination $\mathrm{R} 2=0.47$ and moderate correlation case $(r=0.68)$. The size of neuronal nuclei depended on all modes of irradiation, which had approximately the same effect on the index. The effect of factors on the dynamics of karyometric indicators is average: the level of significance of the model is less than 0.01 with the coefficient of determination R2 $=0.48$ and moderate correlation of arguments $(\mathrm{r}=0.69)$. Protein content in neurons depended on fractionated irradiation, and single irradiation had no significant effect on it. However, the effect of irradiation on the indicator is low: the level of model significance is more than 0.3 with $\mathrm{R} 2=0.14$ determination coefficient and weak correlation of $\mathrm{r}=0.37$ arguments. The content of RNA in the neurons' cytoplasm depended on all modes of irradiation, which equally affect the dynamics of RNA. The effect of irradiation on changes of cytoplasmic RNA is not explicit: the level of significance of the model is less than 0.001 while the coefficient of determination $\mathrm{R} 2=0.69$ and the strong correlation case $(\mathrm{r}=0.83)$.

The content of DNA in the neurons' nuclei depended on all the factors under consideration, but single irradiation had a greater impact. The effect of irradiation on the dynamics of nuclear DNA is quite high: the significance level of the model is less than 0.0001 with the determination coefficient R2 $=0.81$ and strong correlation of arguments ( $\mathrm{r}=0.90)$. The content of RNA in nucleoli depended on all modes of irradiation, but single irradiation (about 2 times) had a greater impact than fractionated irradiation. The effect of irradiation on the dynamics of RNA content in nucleoli is quite high: the significance level of the model is less than 0.0001 with the determination coefficient $\mathrm{R} 2=0.92$ and strong correlation of arguments ( $\mathrm{r}=0.96)$.

Thus, in the long term of the affected period in the III layer of the cortex single irradiation has a greater impact on the dynamics of normochromic neurons, increasing their number and reducing the proportion of destructive neurons. Fractionated irradiation had a greater impact on changes in the functional activity of neurons. In the $\mathrm{V}$ layer of the cortex, single irradiation had a greater impact on the dynamics of normochromic neurons (increasing their number) and on the functional changes of neurons.

The dynamics of destructive neurons was more influenced by fractionated irradiation, increasing their number. Single irradiation had a greater impact on changes in DNA content in nuclei, RNA in nucleoli and cytoplasmic area. Fractionated irradiation was provided either less impact on the indicators under consideration, or approximately equal effect compared with single irradiation. In general, despite a number of features of the dynamics of neuromorphological parameters at single and fractionated irradiation, the studied modes of gamma irradiation at a dose of 50 cGy do not cause significant alterative changes in functionally different layers of the sensory-motor cortex of the cerebral hemispheres. 


\section{ISSN: 2574-1241}

DOI: $10.26717 / B J S T R .2019 .13 .002389$

Ushakov IB. Biomed J Sci \& Tech Res

(C) (i) This work is licensed under Creative

Submission Link: https://biomedres.us/submit-manuscript.php

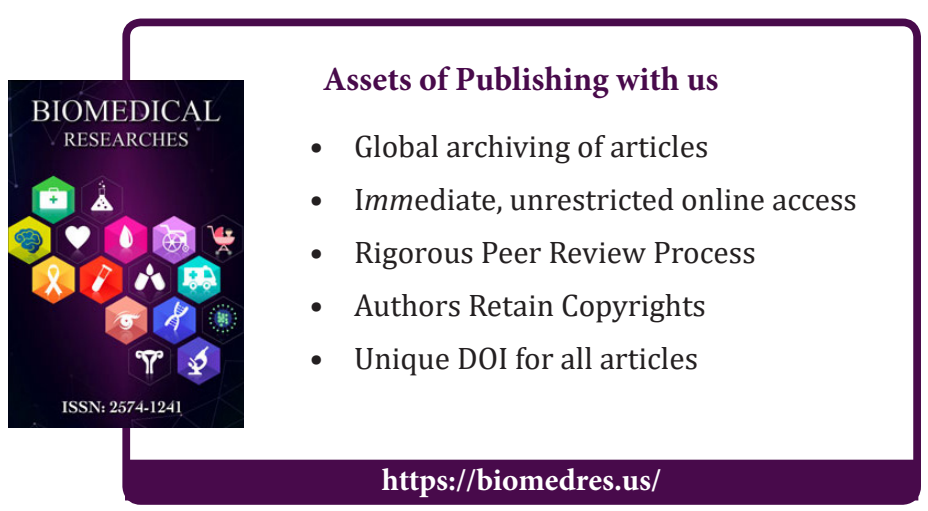

